\title{
Radiation pattern of a planar electromagnetically coupled coaxial dipole array mounted over a finite metallic reflected plate
}

$\operatorname{AUTHOR}(S)$ :

Rao, QJ; Hashiguchi, H; Fukao, S

\section{CITATION:}

Rao, QJ ...[et al]. Radiation pattern of a planar electromagnetically coupled coaxial dipole array mounted over a finite metallic reflected plate. IEEE TRANSACTIONS ON ANTENNAS AND PROPAGATION 2003, 51(5): 1132-1136

ISSUE DATE:

2003-05

URL:

http://hdl.handle.net/2433/50439

\section{RIGHT:}

(c)2003 IEEE. Personal use of this material is permitted. However, permission to reprint/republish this material for advertising or promotional purposes or for creating new collective works for resale or redistribution to servers or lists, or to reuse any copyrighted component of this work in other works must be obtained from the IEEE. 
exists. In addition, the antenna-gain variation in the operating bandwidth is found to be less than $2 \mathrm{dBi}$. Therefore, the proposed design can give a good solution to the problem of tradeoff between the impedance bandwidth and antenna-gain variation for the broadband microstrip antenna with an embedded reactive loading.

\section{REFERENCES}

[1] N. Fayyaz and S. Safavi-Naeini, "Bandwidth enhancement of a rectangular patch antenna by integrated reactive loading," in Proc. IEEE Int. Symp. AP-S, Atlanta, GA, June 21-26, 1998, pp. 1100-1103.

[2] K. L. Wong and J. S. Kou, "Bandwidth enhancement of bow-tie microstrip antenna using integrated reactive loading," Microwave Opt. Technol. Lett., vol. 22, no. 7, pp. 69-71, July 1999.

[3] S. T. Fang, "Analysis and design of triangular microstrip antennas," Ph.D. dissertation, Dept. Elect. Eng., National Sun Yat-Sen Univ., Kaohsiung, Taiwan, R.O.C., June 1999.

[4] K. L. Wong and J. Y. Jan, "Broadband circular microstrip antenna with embedded reactive loading," Electron. Lett., vol. 34, pp. 1804-1805, Sept. 1998.

[5] J. Y. Jan and K. L. Wong, "Microstrip-line-fed broadband circular microstrip antenna with embedded reactive loading," Microw. Opt. Technol. Lett., vol. 22, no. 3, pp. 200-202, Aug. 1999.

\section{Radiation Pattern of a Planar Electromagnetically Coupled Coaxial Dipole Array Mounted Over a Finite Metallic Reflected Plate}

Qinjiang Rao, Hiroyuki Hashiguchi, and Shoichiro Fukao

\begin{abstract}
An efficient numerical method is proposed to analyze the farfield of electromagnetically coupled coaxial dipole (ECCD) element, and then uniform physical theory of diffraction (PTD) together with the above method is applied to investigate the radiation pattern of a novel planar ECCD array mounted over a finite metallic plate. Finally, as an application to the above ECCD array, lower troposphere radar (LTR) is briefly introduced. The analysis procedure in this paper can also be expected for optimum design in the systems involving the ECCD array antenna.
\end{abstract}

Index Terms-Electromagnetically coupled coaxial dipole (ECCD), physical theory of diffraction (PTD), radiation sidelobes.

\section{INTRODUCTION}

The electromagnetically coupled coaxial dipole (ECCD) originally proposed in [1] and [2] is a new type of collinear antenna, it is composed of finite metallic circular pipe acting as radiation element fed electromagnetically by an annular ring slot on the outer conductor of the feeding coaxial cable. Fig. 1(a) shows the geometry of the ECCD array antenna, their collinear arrangement in the axial of coaxial cable with in-phase excitation gives an array performance. As a novel radiation element shown in Fig. 1(b) with structural simplicity, the ECCD has potential for easily constructing a large phased array in many fields, such as wireless communication and atmosphere observations. However, literatures up to now show that available theoretical analysis [1],

Manuscript received April 13, 2001; revised May 13, 2002. The work of Q. Rao was supported by the Japan Society for the Promotion of Science (JSPS) under the Postdoctoral Fellowship for Foreign Researchers under Grant 99235.

The authors are with the Radio Science Center for Space and Atmosphere (RASC), Kyoto University, Kyoto 611-0011, Japan.

Digital Object Identifier 10.1109/TAP.2003.811485

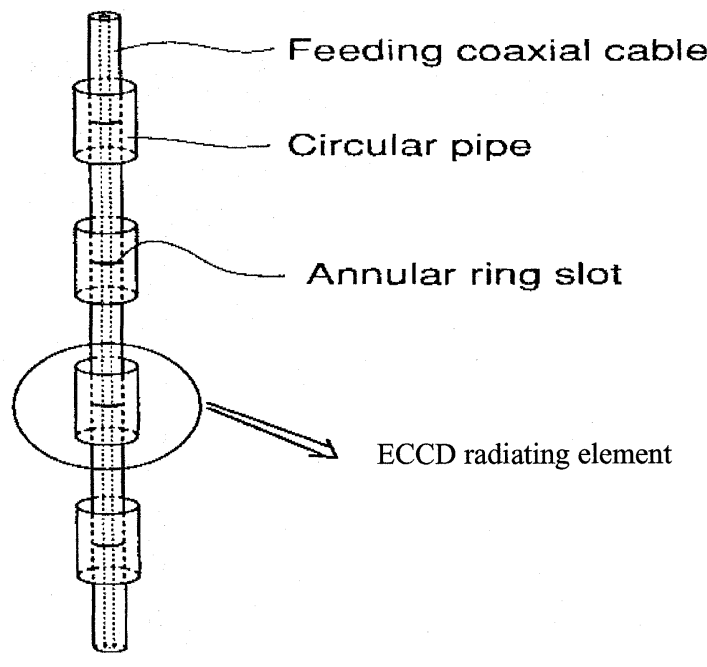

(a)

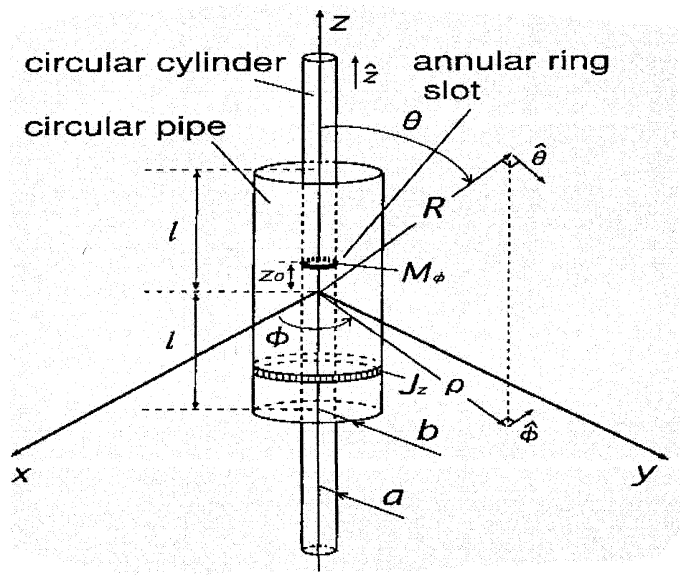

(b)

Fig. 1. (a) Geometry of the ECCD array antenna [1]. (b) Rotationally symmetrical electric and magnetic current on the perfectly finite cylindrical pipe $\boldsymbol{r}$ of an ECCD element [1].

[2] to the ECCD has a disadvantage of practical applications, that is, one must deal with infinite integrals that involve cylindrical functions, and numerical processing is relatively complicated. In addition, the solutions of the above method involves only about one element and a limited linear -4 element array in free space with very electrically small radius and an approximate half-wavelength length for the circular pipe of radiating element. As for its validity for a large array, it has not been certified so far. As a result, practical applications to a large ECCD array have been desiring.

Our first objective in this paper is to apply an alternative numerical method to analyze radiation pattern of the ECCD element. Compared to other method [1], the method in this paper has more abroad suitable ranges for geometry of the circular pipe and has also advantages of more simple and explicit procedure but without loss of accuracy. The basic idea is that magnetic current from the slot on the outer surface of a coaxial cable shown in Fig. 1(b) is interpreted as an incident wave on the circular pipe of the ECCD, and then excites the current distribution on the pipe. This distribution will be determined by applying electric current integration equations. Therefore, the far-field of the induced current on the pipe, known as the scattered field, can be obtained. The secondary objective here is to propose a large 22 by 24 


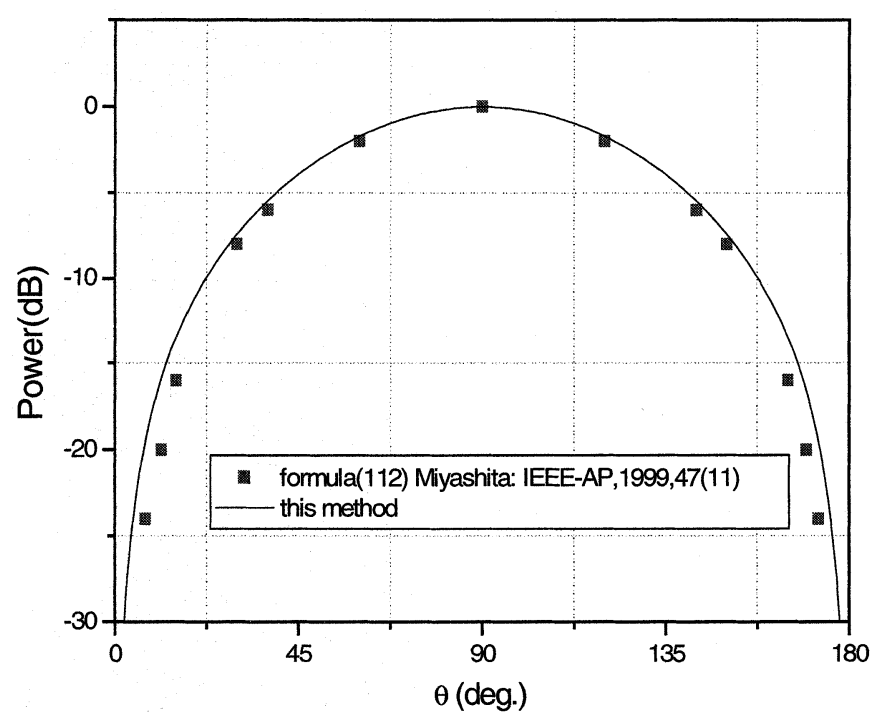

Fig. 2. Comparison of the ECCD radiation patterns simulated by using two kinds of methods. Length of a circular pipe $=\mathbf{0 . 4 2} \boldsymbol{\lambda}_{0}$, diameter of the pipe $=0.46 \lambda_{0}$.

planar ECCD array which is oriented in the horizontal plane, parallel to a finite metallic reflected plate below the array, and then to analyze radiation pattern of the above system by using the uniform physical theory of diffraction (PTD), and furthermore, to investigate the possibility to reduce radiation sidelobes of the ECCD array by adjusting the height of the array over the plate. Finally, based on the above analysis, L band lower troposphere radar (LTR), as a practical application to the ECCD array, is briefly introduced.

\section{ECCD ELEMENT RADIATION FIELD}

As shown in Fig. 1(b), the magnetic current from the slot on the outer conductor of a coaxial cable is interpreted as an incident wave on the circular pipe of the ECCD. The magnetic frill generator was first introduced to calculate the near as well as the far-zone field from a coaxial aperture [3]. Applying this mode, the slot is replaced with a circumferentially directed magnetic current that exits over an annular aperture with inner radius $a$, which is the radius of the outer conductor of the feeding coaxial cable, and outer radius $b$, which is chosen to be the radius of the metallic circular pipe of the ECCD, as shown in Fig. 1. Due to very small radius of the coaxial cable, compared to operating wavelength, the electric field over the annular aperture of the magnetic frill generator can be represented by

$$
E_{f}=\vec{a}_{\rho} \frac{V_{i}}{2 \rho \ln (b / a)} \quad(a \leq \rho \leq b)
$$

the corresponding equivalent magnetic current density $M_{f}$ for the magnetic frill generator used to represent the aperture is equal to

$$
M_{f}=-2 \vec{n} \times E_{f}=-\vec{a}_{\phi} \frac{V_{i}}{\rho^{\prime} \ln (b / a)} \quad(a \leq \rho \leq b) .
$$

The near-field generated by the magnetic frill generator on the surface of the metallic circular pipe is found by [3]

$$
\begin{aligned}
& E_{z}^{i} \approx-V_{i}\left(\frac{k\left(a^{2}-b^{2}\right) e^{-j k R_{0}}}{8 \ln (b / a) R_{0}^{2}}\right. \\
&\left.\cdot\left\{2\left[\frac{1}{k R_{0}}+j\left(1-\frac{a^{2}-b^{2}}{2 R_{0}^{2}}\right)\right]+\cdots\right\}\right)
\end{aligned}
$$

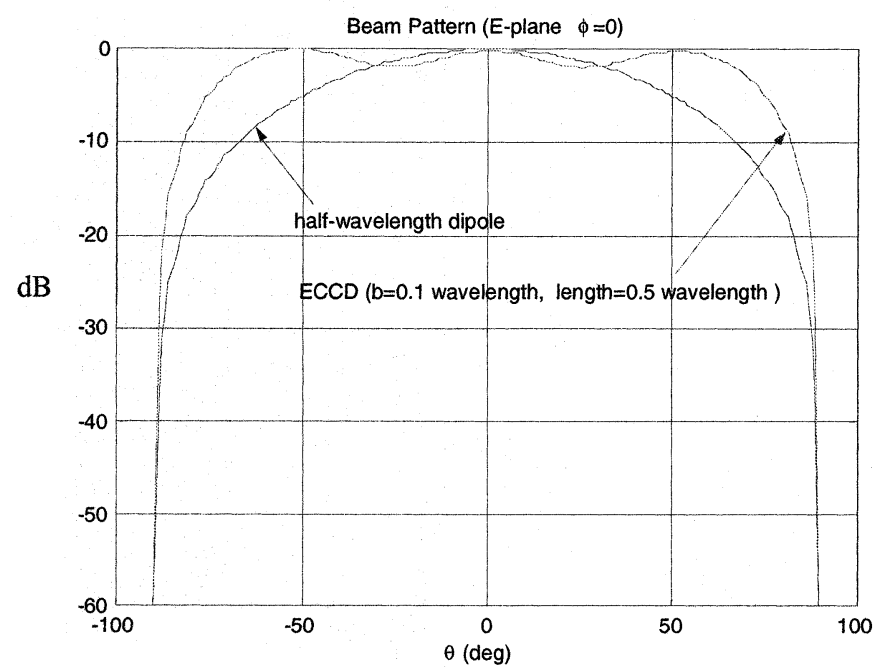

(a)

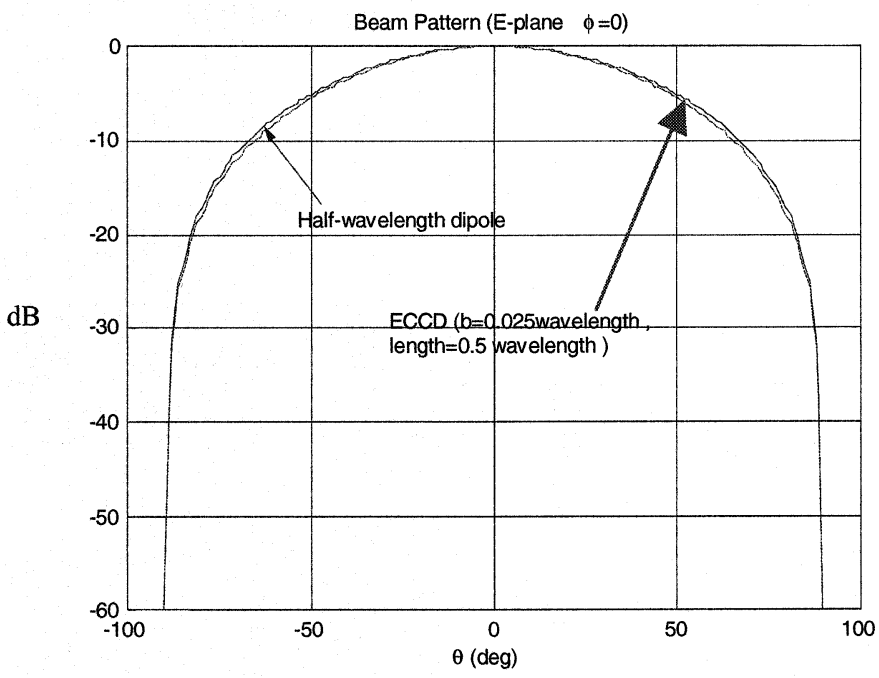

(b)

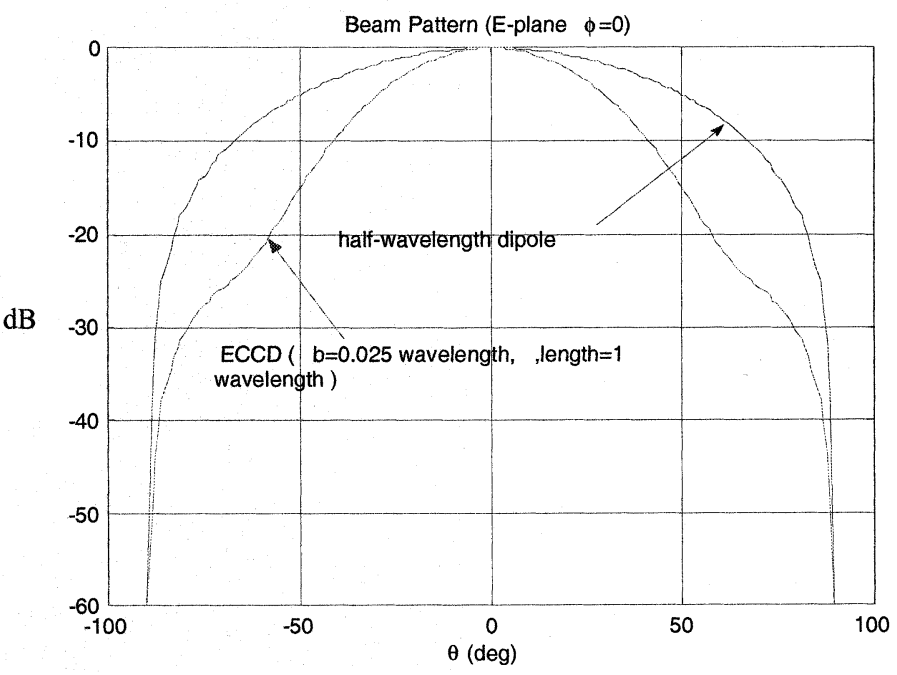

(c)

Fig. 3. (a) Comparison of radiation patterns between ECCD ( $\boldsymbol{a}=$ $\mathbf{0 . 0 0 1} \boldsymbol{\lambda}_{0}$ ) and a half-wavelength dipole. (b) Comparison of radiation patterns between ECCD $\left(\boldsymbol{a}=\mathbf{0 . 0 0 1} \boldsymbol{\lambda}_{0}\right)$ and a half wavelength dipole. (c) Comparison of radiation patterns between ECCD $\left(\boldsymbol{a}=\mathbf{0 . 0 0 1} \boldsymbol{\lambda}_{0}\right)$ and a half wavelength dipole. 


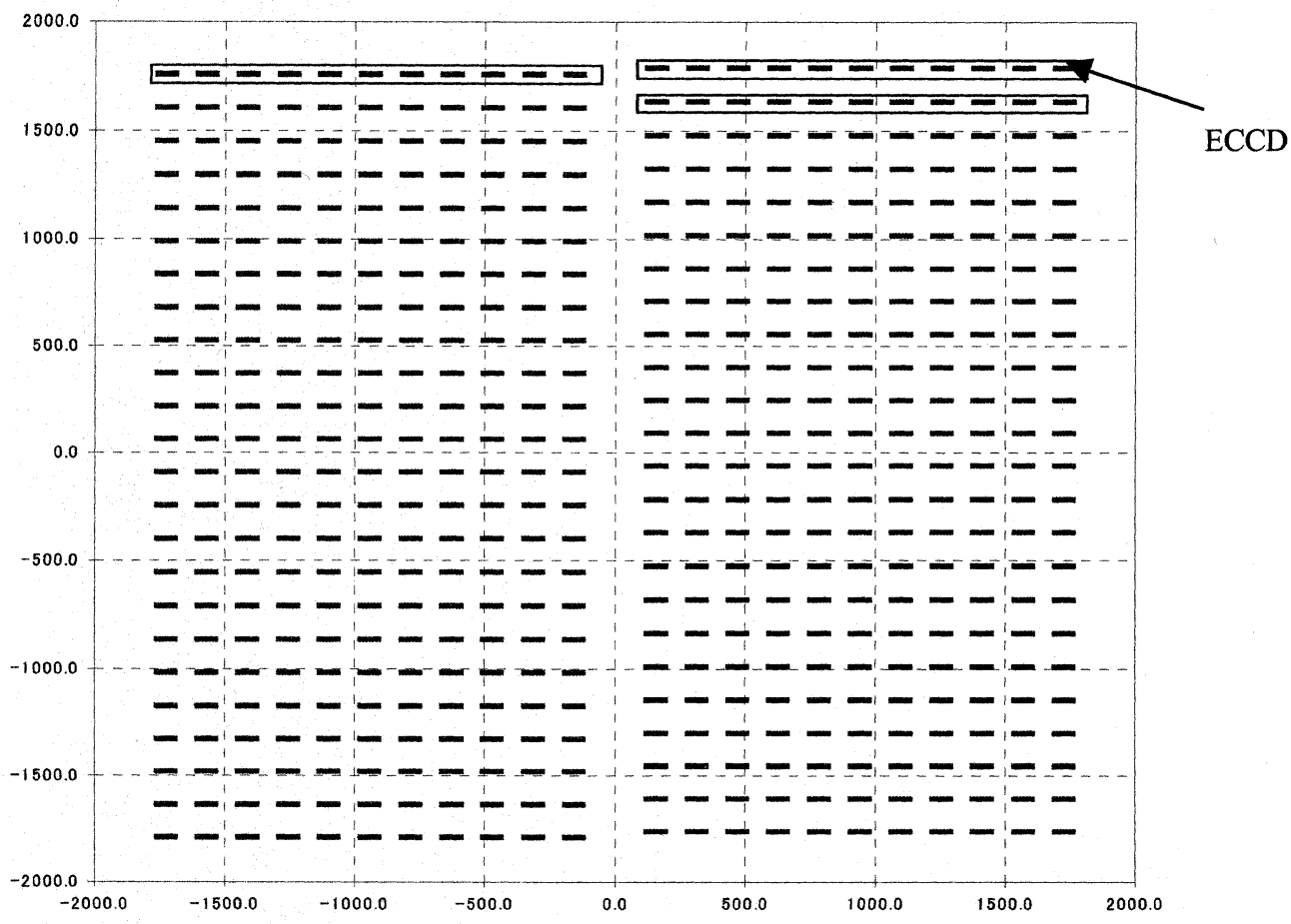

Fig. 4. Element arrangement of a 22 by 24 planar ECCD array (unit: millimeters).

$$
\begin{aligned}
& +\frac{b^{2}}{R}\left[\left(\frac{1}{k R_{0}}+j\left(1-\frac{\left(a^{2}+b^{2}\right)}{2 R_{0}^{2}}\right)\right)\left(-j k-\frac{2}{R_{0}}\right)+\cdots\right] \\
& +\left(-\frac{1}{k R_{0}^{2}}+j \frac{a^{2}+b^{2}}{R_{0}^{2}}\right)
\end{aligned}
$$

where $R_{0}=\sqrt{z^{2}+a^{2}}$

Let an incident wave $E^{i}(r)$ impinge on the circular pipe shown in Fig. 1(b), and induce a linear current density $J_{s}$ on the surface, the induced current re-radiates and produces an electric field $E^{s}(r)$, therefore, the total field $E^{t}(r)$ in space is the sum of the incident and the scattered field

$$
E^{t}(r)=E^{i}(r)+E^{s}(r) .
$$

If the observation point is moved to the metallic pipe, the total tangential electric field vanishes for perfectly conducting surface.

To obtain current distribution, we introduce a current integration equation

$$
\int_{-l}^{l} I_{z}\left(z^{\prime}\right)\left[\left(\frac{\partial^{2}}{\partial z^{2}}+k^{2}\right) G\left(z, z^{\prime}\right)\right] d z=-i \omega \varepsilon E^{i} \quad(\rho=b)
$$

where

$$
\begin{aligned}
G\left(z, z^{\prime}\right) & =\frac{1}{2 \pi} \int_{0}^{2 \pi} \frac{e^{-j k R}}{4 \pi R} d \phi^{\prime} \\
R & =\sqrt{4 b^{2} \sin ^{2}\left(\frac{\phi^{\prime}}{2}\right)+\left(z-z^{\prime}\right)^{2}} .
\end{aligned}
$$

$E^{i}$ is given by (3). Once the current contribution on the circular pipe of the ECCD is known, radiating electronic field can be obtained by

$$
\begin{aligned}
& E=-j \omega A-j \frac{1}{\omega \mu \varepsilon} \nabla(\nabla \cdot A) \\
& A=\frac{\mu}{4 \pi} \iint_{s} J_{s}\left(x^{\prime}, y^{\prime}, z^{\prime}\right) \frac{e^{-j k R}}{R} d s^{\prime}
\end{aligned}
$$

for the far-field

$$
E \approx-j \omega A
$$

where $k=\omega^{2} \mu \varepsilon, \mu$ is permeability, $\varepsilon$ is dielectric constant, $R$ is the distance from any point in the source to the observation point.

In order to check the above procedures, we compare the ECCD element patterns obtained by using the above method and other method, respectively. Fig. 3 shows a good agreement. To further illustrate the influence of the geometry of circular pipe of the ECCD on its radiation pattern, we also simulate radiation patterns of the ECCD with different circular radii and lengths. It appear from Fig. 3(a)-(c) that radiation pattern of an ECCD element is fully identical to that of a half wavelength dipole if the radius of its circular pipe is less than 0.025 wavelength and its length is equal to 0.5 wavelength.

\section{Field Scattered by a Finite Metallic Plate Mounted BELOW THE ECCD PHASED ARRAY}

In this section, a planar 22 by 24 ECCD array is introduced as shown in Fig. 4 where each array is composed of twenty-four rows of ECCD linear array, and each row includes 22 basic ECCD radiation elements, each row is further subdivided into two subelements. By controlling the phase of the phase shifter, we can steer the beam in the different direction.

To reduce low elevation sidelobes, we propose a finite metallic reflected plate parallel to and under the array. Due to edge diffraction from a finite plate, we will see that it is crucial how to select suitable array height over the plate.

Uniform PTD technique is used to investigate the field scattered by a finite metallic plate mounted below the ECCD array. The total field in space can be obtained [4]

$$
\begin{aligned}
E & =E_{\mathrm{PO}}+\left(E_{\mathrm{UTD}}^{d}-E_{\mathrm{POF}}^{d}\right)=E_{\mathrm{PO}}+E_{\mathrm{PTD}}^{d} \\
E_{\mathrm{PO}} & =E^{i}+E_{\mathrm{PO}}^{s} \\
E_{\mathrm{PO}}^{s} & =2 \iint_{s}\left(\vec{n} \times H^{i}\right) d s
\end{aligned}
$$




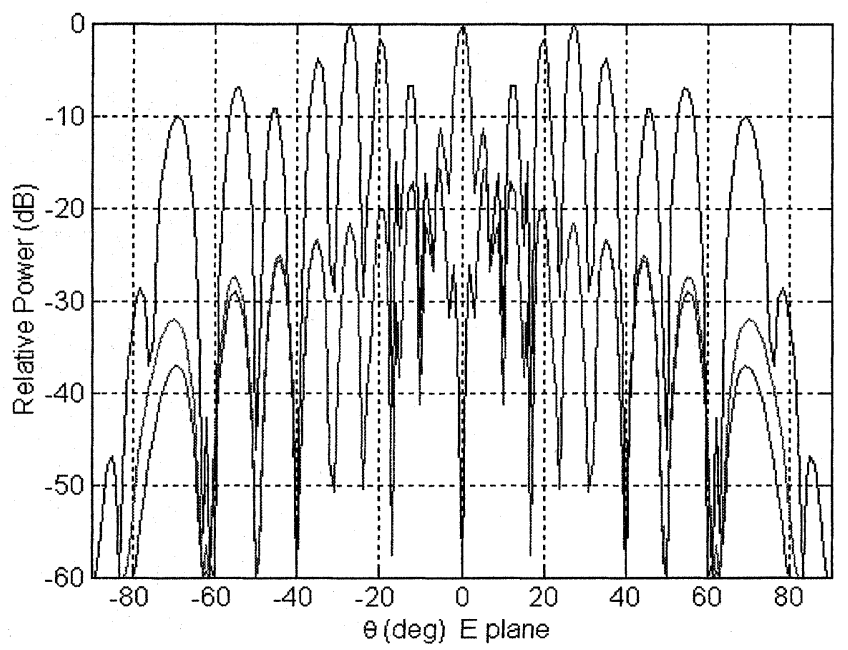

(a)

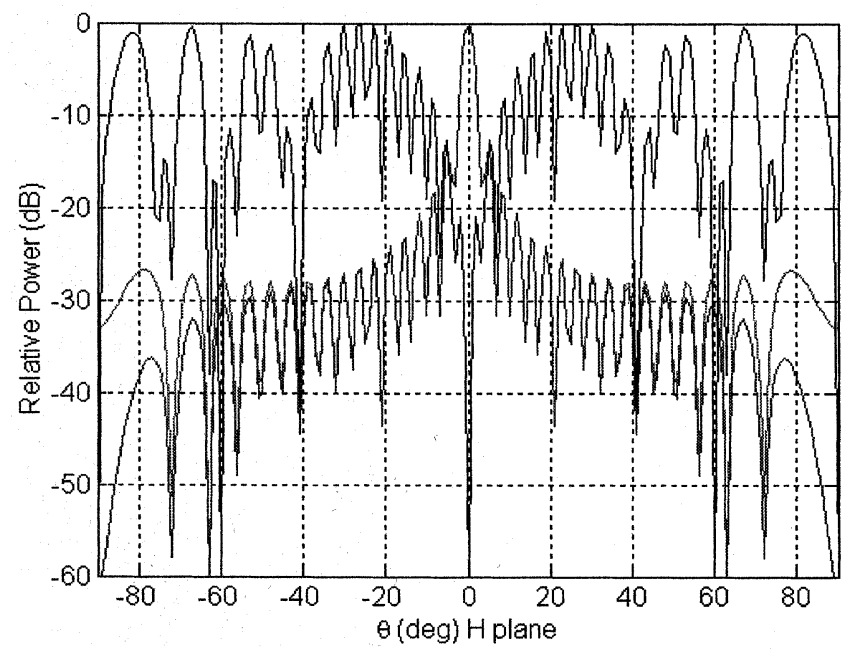

(b)

Fig. 5. (a) Effects of height of a 22 by $24 \operatorname{ECCD}\left(\boldsymbol{b}=\mathbf{0 . 0 2 5} \boldsymbol{\lambda}_{0}, \boldsymbol{a}=\right.$ $0.009 \lambda_{0}$, length $=0.5 \boldsymbol{\lambda}_{0}$ ) array over a square plate on radiation pattern. The sidelength of the plate is $18.1818 \boldsymbol{\lambda}_{0}$. The height of the array over the plate is $0.25 \boldsymbol{\lambda}_{0}$ and $2 \boldsymbol{\lambda}_{0}$ for bottom line and top line, respectively. There is no plate for middle line.

where $s$ is surface area of a scatterer, $E^{i}$ is the total radiation field contributed by every array element, it can be given by using superposition approach after neglecting mutual coupling among elements due to the beam directed broadside. $E_{\mathrm{PO}}$ is physical optics field, $E_{\mathrm{UTD}}^{d}$ and $E_{\mathrm{POF}}^{d}$ is diffraction field of uniform geometry optics and uniform physics optics due to finite edge, $E_{\mathrm{PTD}}^{d}$ is the total diffraction field in PTD, the general definition and related formula for the above parameters can also be found from [5]. But it should be noted that the stationary phased points along the four edges of the plate are just chosen as diffraction points for calculating diffraction fields in the two principal planes, namely $E$ and $H$.

As PO field, let the plate to be divided into $N$ segments and the center of the $i$ th segment to be located at $\left(x_{i}, y_{i}, z_{i}\right)$, and then the total field at the $i$ th segment can be obtained by

$$
H^{i}=\sum_{k=1}^{M} H_{k}^{i}\left(x_{i}, y_{i}, z_{i}\right)=\sum_{k=1}^{M}\left(-\frac{1}{j \omega \mu} \nabla \times E_{k}^{i}\right)
$$

where $E_{k}^{i}$ is the electric field at the center of the $i$ th segment due to the $k$ th element in the array which is obtained from (9), $M$ is the total

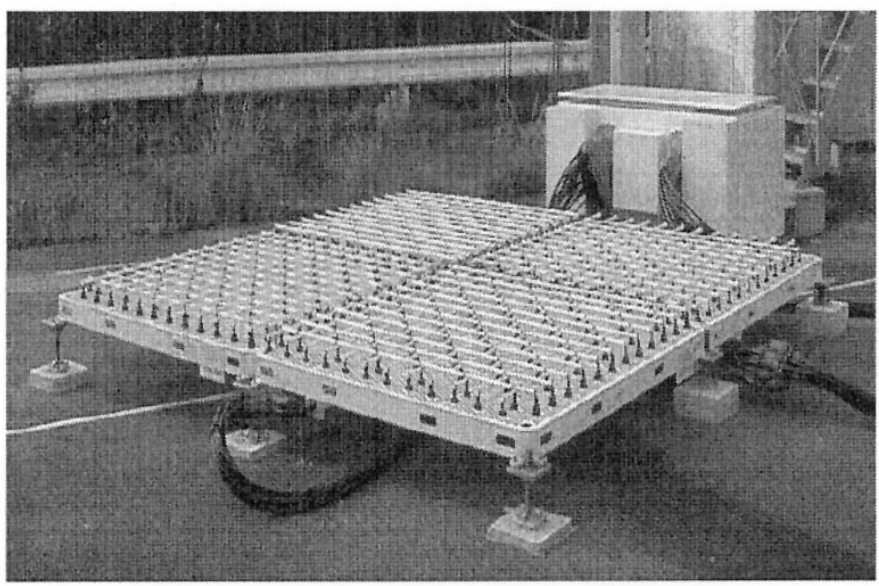

(a)

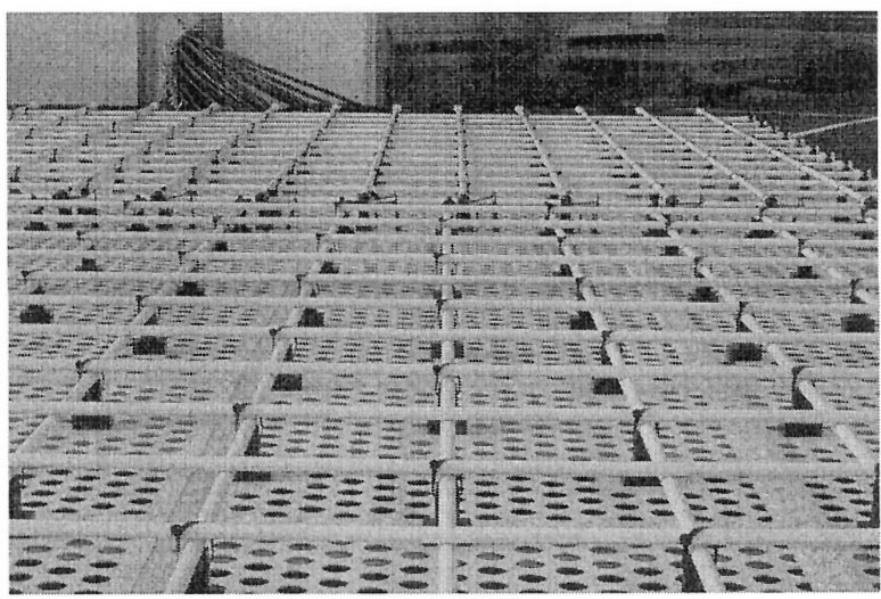

(b)

Fig. 6. (a) L band LTR at the Shigaraki MU Observatory of Kyoto University. (b) Two perpendicular 22 by 24 ECCD $\left(\boldsymbol{b}=\mathbf{0 . 0 2 5} \boldsymbol{\lambda}_{0}, \boldsymbol{a}=\mathbf{0 . 0 0 9} \boldsymbol{\lambda}_{0}\right.$, length $=0.5 \boldsymbol{\lambda}_{0}$ ) array mounted $0.25 \boldsymbol{\lambda}_{0}$ over a reflected plate of $4 \mathrm{~m}$ by $4 \mathrm{~m}$ $\left(18.1818 \boldsymbol{\lambda}_{0}\right)$, the very small holes are just for drainage.

number of array elements. Therefore, the total current on the $i$ th segment can be written as the following:

$$
\begin{aligned}
J_{\mathrm{PO}}(i) & =2\left(\vec{n}_{i} \times H^{i}\right)=-\frac{2}{j \omega \mu} \sum_{k=1}^{M} \vec{n}_{i} \times \nabla \times E_{k}^{i} \\
& \quad \text { for illuminated parts of the surface } \\
& =0, \quad \text { for shadow parts }
\end{aligned}
$$

where $\vec{n}_{i}$ is an unit outer normal vector of the $i$ th segment. After knowing the induced current on the $i$ th segment, the total PO scattered field in space contributed by $N$ segments is therefore given by

$$
\begin{aligned}
& E_{\theta}^{s}(\text { total })=\sum_{i=1}^{N} E_{\theta}^{s}(i)=-\frac{j \eta k e^{-j k R}}{4 \pi R} \\
& \sum_{i=1}^{N} \Delta s_{i} J_{\mathrm{PO}}(i) e^{-j k\left(x_{i} \sin \theta \cos \phi+y_{i} \sin \theta \sin \phi+z_{i} \cos \theta\right)} .
\end{aligned}
$$

\section{Modeling RESUltS AND APPLICATION}

In simulation samples, we adopt a 22 by 24 ECCD array and all element spacing is $0.70 \lambda_{0}, \lambda_{0}$ is operating wavelength in free space. Four diffraction points, located at in $E$ and $H$ principal planes, are 
selected for simulating diffraction fields since the geometry of the plate and the ECCD array is symmetrical.

We investigate the possibility to reduce sidelobes of the ECCD array by mounting a finite reflected plate below the array. It is well known that dipoles with infinite ground plane can achieve the maximum gain if the height of dipoles over the ground is 0.25 wavelength. Extensive calculations in this paper have indicated that a finite reflected plate with an appropriate size can also achieve the same effects for increasing gain and reducing sidelobes as an infinite plate. It has been observed from Fig. 5 that the height of 0.25 wavelengths actually achieves a greatly reduced sidelobes as well as the maximum gain. This is because the diffraction by finite edges of the plate is quite small, compared to reflection from the surface of plate. The total scattering field over the above half-space of a finite plate is almost equal to the sum of two parts, one is from the direct radiation of antennas and the other from full reflection due to the ground plate. The above results are just desired for some radars to increase gain and to block ground clutter signals.

Based on the above simulations, we adopt a 22 by 24 planar ECCD array to the LTR shown in Fig. 6. The LTR is a 1.36-GHz pulse-modulated monostatic Doppler radar with an active phased array system, where the antennas consists of two rectangular arrays, they are perpendicularly superimposed corresponding to two linear polarization. The configuration of each array is the same as that mentioned in Section III. The nominal peak power is $2 \mathrm{~kW}$ (the maximum average power is 400 $\mathrm{W}$ ), which is produced by 24 solid state power amplifiers (transmitter modules). The LTR has been successfully operated at the Shigaraki MU radar observatory of Kyoto University for the lower atmosphere observation.

\section{Conclusion}

An efficient analysis procedure is presented for exactly modeling the far field of ECCD element. One of its advantages is that one need not handle infinite integrals raised [1] for the process is based on Pocklington type of formulation that only involves closed form of Green's functions in free space. This allows the proposed analysis process in this paper to be easily applied to various ECCD elements with different lengths and radii of circular pipe. Owing to its advantages of simply constructed and clear physical meanings, the procedure in this paper, compared to available methods from other literatures, can also be more easily applied in engineering.

The equivalent model derived from the analysis for the ECCD element can result in the simplification for the computation of the radiation pattern of the ECCD under some special conditions, and therefore can increase computation efficiency.

It is fully novel configuration in the ECCD application to mount a large ECCD planar array over a finite metallic reflected plate with a suitable height so as to achieve reduced radiation sidelobes of array antenna. The uniform physical theory of diffraction, being hybrid with the proposed method for the ECCD element, has handled successfully the scattered far field of this system, the proposed height for ECCD array antenna over the plate is practical useful.

The proposed ECCD array configuration in the LTR is only a start for ECCD array application. Authors believe that the related configuration can also be expected to application in other fields, such as based station antennas for wireless communications.

\section{ACKNOWLEDGMENT}

This work has benefited from the input of Mitsubishi Electric Corporation (MELCO), Kanagawa, Japan. The authors are grateful for useful discussions with Dr. Miyashita of MELCO.

\section{REFERENCES}

[1] H. Miyashita, H. Ohmine, K. Nishizawa, S. Makino, and S. Urasaki, "Electromagnetically coupled coaxial dipole array antenna," IEEE Trans. Antennas Propagat., vol. 47, no. 11, pp. 1716-1725, Nov. 1999.

[2] H. Miyashita, "Study on analytical modeling of anatenna arrays for implementation of efficient design procedure," Ph.D. dissertation, Radio Science Center for Space and Atmosphere, Kyoto University, ch. 2, May 2000.

[3] L. L. Tsai, "A numerical solution for the near and far fields of an annular ring of magnetic current," IEEE Trans. Antennas Propagat., vol. AP-20, pp. 569-576, Sept. 1972.

[4] E. Yamashita, Analysis Methods for Electromagnetic Wave Problems. Norwood, MA: Artech House, 1996, Physical Optics by Makoto Ando, ch. 4.

[5] A. K. Bhattacharyya, High Frequency Electromagnetic Techniques: Recent Advances and Applications. New York: Wiley, 1995.

\section{Radar Cross Section of Stacked Circular Microstrip Patches on Anisotropic and Chiral Substrates}

\author{
V. Losada, R. R. Boix, and F. Medina
}

\begin{abstract}
Galerkin's method in the Hankel transform domain (HTD) is applied to the determination of the radar cross section (RCS) of stacked circular microstrip patches fabricated on a two-layered substrate which may be made of a uniaxial anisotropic dielectric, a magnetized ferrite or a chiral material. Concerning the case of stacked patches printed on magnetized ferrites, the results show that substantial RCS reduction can be achieved inside the tunable frequency band where magnetostatic mode propagation is allowed. It is also shown that both the frequency and the level of the RCS peaks obtained for circular patches fabricated on anisotropic dielectrics or chiral materials may be substantially different from those obtained when substrate anisotropy or substrate chirality are ignored.
\end{abstract}

Index Terms-Anisotropic media, chiral media, ferrites, microstrip antennas, scattering.

\section{INTRODUCTION}

Although the standard configuration for a microstrip antenna involves one single conductor patch, two stacked conductor patches are sometimes employed for obtaining either dual-frequency operation [1] or increased bandwidth operation [1], [2]. Concerning the topic of scattering from microstrip antennas, we should say that as the radar cross section (RCS) of military platforms is reduced by geometrical shaping and the use of composite radar absorbing materials, the scattering from the antennas mounted on such structures may give the most important contribution to the overall RCS. Bearing in mind that microstrip antennas are very suitable for use on aircraft vehicles owing to their light weight and conformability, different results were published in the past for the RCS of single microstrip antennas both on conventional isotropic dielectric substrates [3] and nonconventional substrates with dielectric and magnetic anisotropy [4], [5]. The feeding and radiation

Manuscript received September 28, 2001; revised January 28, 2002. This work was supported by the El Centro de Investigación Científica y Tecnológica (CICYT), Spain under Grant TIC98-0630.

V. Losada is with the Microwaves Group, Department of Applied Physics, E.U.I.T.A., University of Seville, 41013, Sevilla, Spain.

R. R. Boix and F. Medina are with the Microwaves Group, Department of Electronics and Electromagnetism, College of Physics, University of Seville, 41012 Sevilla, Spain (e-mails: boix@us.es; medina@us.es).

Digital Object Identifier 10.1109/TAP.2003.811528 